\title{
Free Convection Fluid Flow from a Spinning Sphere with Temperature- Dependent Physical Properties
}

\author{
Gilbert Makanda \\ Department of Mathematical and Physical Sciences, Central University of Technology \\ Free State, Private Bag X20539, Bloemfontein, 9300, South Africa \\ gmakanda@cut.ac.za
}

\begin{abstract}
Natural convection from a spinning sphere with temperature dependent viscosity, thermal conductivity and viscous dissipation was studied. A unique system of non-similar partial differential equations was solved using the bivariate local-linearization method (BLLM). This method use Chebyshev spectral collocation method applied in both the $\eta$ and $\xi$ directions. Similar equations in the literature are normally solved by inaccurate time-consuming finite difference methods. This work introduces a robust method for solving partial differential equations arising in heat and mass transfer. The numerical method was validated by comparison to the results previously published in the literature. The method is fully described in this article and can be used as an alternative method in solving boundary value problems. This work also presents rarely reported results of the effect of selected parameters on spin-velocity profiles $g(\eta)$.
\end{abstract}

Keywords: Free convection, spinning sphere, local linearization

\section{Introduction}

The concept of free convection from spherical surfaces has received attention in the area of fluid flow due to its practical application. These applications include among others studies around the earth's atmosphere, cooling around spherical surfaces. These studies applied numerical solutions to ordinary and partial differential equations but did not focus on the accuracy of the numerical methods. We discuss the methods used in the literature and new method introduced in this work. These methods are finite differences; implicit and explicit, the Keller-box, the Homotopy analysis method, the local non-similarity method of Minkowycz and Sparrow [1], Runge- Kutta method. In this work we discuss the newly developed bivariate local linearization method (BLLM) in Motsa et. al [2]. The method was extended from the quasi-linearization method (QLM) of Bellman and Kalaba [3]

It is well known that analytical methods are restricted to simple cases and equations which normally do not accurately describe practical situations. We therefore make use of numerical methods. Numerical methods have been widely reported to be accurate and sometimes are comparable to analytical solutions. The methods include finite difference techniques reported by Gaffar et. al. [4], El-Amin and Sun [5], Ramteke and Kishore [6], Rhahimi and Jalali [7] and Zainuddin et al. [8]. The finite difference based Kellerbox method is also reported by Blottner et al. [9], Nayela et. al. [10], Haquea et. al [11], Alim et. al [12] and Narayana et. al. [13]. These methods have widely been used, they involve approximating functions and their derivatives in differential equations. These methods are accurate but need more computation time.

In numerical methods, efforts have been made to reduce computational time and achieve accurate results. More accurate hybrid methods were developed, such as the Homotopy analysis method which was reported in Makukula et. al [14], Motsa and Sibanda [15] and Nadeem and Saleem [16]. This method is easy to implement and accurate and better than finite difference methods. The solution of ordinary differential equations arising in fluid flow were also solved using Matlab bvp4c based on Runge-Kutta method. The method is adaptive (adjusts) the numerical step size at every iteration Bogacki and Shampine [17]. The Runge-Kutta method is easy to use and more accurate than the traditional finite difference methods, Shampine [18]. The Runge-Kutta method was used by among others Ghalambaz et. al. [19] and Kameswaran et. al. [20].

This study focuses on the application of more accurate numerical method based on spectral collocation technique to a system of partial differential equations arising in fluid flow. Fluid flow around spheres was studied by among others Kabeir et. al. [21], Jafarpur and Yavanovich [22] and Nayela et. al. [10], Gaffar et. al. [4], in their work they applied finite 
difference techniques such as the Keller-box method. In this work we show that the bivariate local linearization method (BLLM) can be used as an alternative method for solving boundary value problems accurately. The method is easy to implement as shown in the solution method section.

\section{Mathematical Formulation}

Consider a steady, incompressible three dimensional flow of a Newtonian fluid around a sphere. The Cartesian coordinates $\mathrm{x}, \mathrm{y}$ and $\mathrm{z}$ are along the, $\mathrm{u}, \mathrm{v}$ and $\mathrm{w}$ are the respective velocity components. The surface of the sphere is maintained at constant temperature $T_{s}$ and the ambient temperature at $T_{\infty}$.

The governing equations can be written as:

$$
\begin{gathered}
\frac{\partial(a u)}{\partial x}+\frac{\partial(a v)}{\partial y}=0 \\
u \frac{\partial u}{\partial x}+v \frac{\partial u}{\partial y}-\frac{w^{2}}{x}=\frac{\mu(T)}{\rho} \frac{\partial^{2} u}{\partial y^{2}}-\frac{\mu(T)}{\rho} u+\frac{1}{\rho} \frac{\partial \mu(T)}{\partial T} \frac{\partial T}{\partial y} \frac{\partial u}{\partial y}+g \beta_{T}\left(T-T_{\infty}\right) \sin \frac{x}{a} \\
u \frac{\partial w}{\partial x}+v \frac{\partial w}{\partial y}-\frac{u w}{x}=\frac{\mu(T)}{\rho} \frac{\partial^{2} w}{\partial y^{2}}-\frac{\mu(T)}{\rho} w \\
u \frac{\partial T}{\partial x}+v \frac{\partial T}{\partial y}=\frac{\kappa(T)}{\rho C_{p}} \frac{\partial^{2} T}{\partial y^{2}}+\frac{1}{\rho C_{p}} \frac{\partial \kappa(T)}{\partial T}\left(\frac{\partial T}{\partial y}\right)^{2}+\frac{\mu(T)}{\rho C_{p}}\left[\left(\frac{\partial u}{\partial y}\right)^{2}+\left(\frac{\partial w}{\partial y}\right)^{2}\right]
\end{gathered}
$$

Equations (1) - (4) are subject to boundary conditions

$$
\begin{gathered}
u=0, v=-V_{s}, w=a \Omega, T=T_{s}, y=0, \\
u \rightarrow 0, w \rightarrow 0, T \rightarrow T_{\infty}, y \rightarrow \infty,
\end{gathered}
$$

where the subscripts $s$ and $\infty$ refer to the surface and ambient conditions. a is the radius of the sphere, $r$ is the density of the fluid, $K$ is permeability parameter, $C_{p}$ is the specific heat capacity, $g$ is the acceleration due to gravity, $b_{T}$ is the coefficient of thermal expansion of the fluid, $V_{s}$ is the suction velocity at the surface and, $\Omega$ is the angular velocity of the spinning sphere.

We assume linear surface temperature on the sphere surface. The dynamic viscosity and thermal conductivity varies as a linear function of temperature as in Animasaun [23].

$$
\begin{gathered}
\mu(T)=\mu_{0}\left[a_{1}+b_{1}\left(T_{s}-T\right)\right] \\
\kappa(T)=\kappa_{0}\left[a_{2}+b_{2}\left(T-T_{\infty}\right)\right]
\end{gathered}
$$

where $\mu_{0}$ is the coefficient of viscosity and $\kappa_{0}$ is the constant value of the coefficient of thermal conductivity further away from the sphere surface, $A, a_{1}, a_{2}, b_{1}, b_{2}$ are constants; in this study we consider $a_{1}=a_{2}=1$ only,

Using the following similarity transformations

$$
\xi=\frac{x}{a}, \eta=\frac{y}{a} G r^{\frac{1}{2}}, \psi=v_{0} \xi G r^{\frac{1}{4}} f(\xi, \eta)
$$




$$
\begin{gathered}
u=\frac{v_{0} G r^{\frac{1}{2}}}{a} \xi f^{\prime}(\xi, \eta), v=-\frac{v_{0} G r^{\frac{1}{4}}}{a}\left[\xi \frac{\partial f}{\partial \xi}-(1+\xi \cot \xi) f\right] \\
w=\frac{v_{0} G r^{\frac{3}{4}}}{a} g(\xi, \eta), \theta=\frac{T-T_{\infty}}{T_{s}-T_{\infty}}
\end{gathered}
$$

Where $\mathrm{Gr}=\left(\mathrm{gb}_{\mathrm{T}}\left(\mathrm{T}_{\mathrm{s}}-\mathrm{T}_{\infty}\right) \mathrm{a}^{3}\right) / \mathrm{u}_{0}{ }^{2}$ is the Grashof number and $\psi$ is the dimensional stream function defined by $\mathrm{au}=\partial \mathrm{y} / \partial \mathrm{y}$ and $\mathrm{av}=-\partial \mathrm{y} / \partial \mathrm{x} \cdot \mathrm{u}_{0}=\mathrm{m}_{\mathrm{b}} / \mathrm{r} v_{0}$ is the constant kinematic viscosity of the fluid. we obtain the following partial differential equations are written as

$$
\begin{gathered}
\left(1+\epsilon_{1}(1-\theta)\right) f^{\prime \prime \prime}+2(1+\xi \cot \xi) f f^{\prime \prime}-f^{\prime 2}-\epsilon g^{2}-\epsilon_{1} \theta^{\prime} f^{\prime \prime} \\
+K_{p}\left[1+\epsilon_{1}(1-\theta)\right] f^{\prime}+\theta=\xi\left(f^{\prime} \frac{\partial f^{\prime}}{\partial \xi}-f^{\prime \prime} \frac{\partial f}{\partial \xi}\right) \\
\left(1+\epsilon_{1}(1-\theta)\right) g^{\prime \prime}+(1+\xi \cot \xi) f g^{\prime}-2 f^{\prime} g-K_{p}\left[1+\epsilon_{1}(1-\theta)\right] g=\xi\left(f^{\prime} \frac{\partial g}{\partial \xi}-g^{\prime} \frac{\partial f}{\partial \xi}\right) \\
\left(1+\epsilon_{2} \theta\right) \theta^{\prime \prime}+\operatorname{Pr}\left(f \theta^{\prime}-f^{\prime} \theta\right)+\epsilon_{2} \theta^{2}+(1+\xi \cot \xi) f \theta^{\prime} \\
+\operatorname{PrEc}\left[1+\epsilon_{1}(1-\theta)\right]\left(f^{\prime \prime 2}+g^{\prime 2}\right)=\xi\left(f^{\prime} \frac{\partial g}{\partial \xi}-g^{\prime} \frac{\partial f}{\partial \xi}\right)
\end{gathered}
$$

and the boundary conditions are written as

$$
\begin{gathered}
f^{\prime}=0, \xi \frac{\partial f}{\partial \xi}+(1+\xi \cot \xi) f=0, g=1, \theta=1, \quad \eta=0 \\
f^{\prime} \rightarrow 0, g \rightarrow 0, \theta \rightarrow 1, \quad \eta \rightarrow \infty
\end{gathered}
$$

where 'prime' denotes the differentiation with respect to $\eta, \varepsilon$ is the spin parameter, $\varepsilon_{1}$ is the temperature-dependent

viscosity parameter, $\varepsilon_{2}$, is the thermal conductivity parameter, $E c$ is the Eckert number, $\mathrm{K}_{\mathrm{p}}=1 / \mathrm{Gr}^{\frac{1}{2}} \mathrm{Da}$ is the Darcian drag force term and $P r$ is the Prandtl number.

\section{Solution Method}

The resulting differential equations were solved using the bivariate local-linearization method (BLLM). The iteration scheme for solving the coupled non-linear partial differential equations system (12)- (14)

$$
\aleph_{k}[F, G, T]=0, k=1,2,3 .
$$

where $\aleph_{1}, \aleph_{2}$ and $\aleph_{3}$ are non-linear operators that denote equations (12) -(14) respectively. $F$, $G$ and $T$ are defined as

$$
F=\left(f, f^{\prime}, f^{\prime \prime}, f^{\prime \prime \prime}, \frac{\partial f}{\partial \xi}, \frac{\partial f^{\prime}}{\partial \xi}\right), G=\left(g, g^{\prime}, g^{\prime \prime}, \frac{\partial g}{\partial \xi}\right), T=\left(\theta, \theta^{\prime}, \theta^{\prime \prime}, \frac{\partial \theta}{\partial \xi}\right)
$$


where primes are the derivatives with respect to $h$. The quasi-linearization method which is based on the Taylor series expansion of $\aleph_{k}$ about some previous approximation of the solution denoted by $\left[F_{r}, G_{r}, T_{r}\right]$, It is assumed that the difference between the current and previous solution is small. Applying the quasi-linearization on equations (12) -(14) gives

$$
\aleph_{k}[F, G, T] \approx \aleph_{k}\left[F_{r}, G_{r}, T_{r}\right]+\left[F-F_{r}, G-G_{r}, T-T_{r}\right] . \nabla \aleph_{k}\left[F_{r}, G_{r}, T_{r}\right]
$$

where $\nabla$ is a vector of partial derivatives defined as $\nabla=\left\{\nabla_{\mathrm{f}}, \nabla_{\mathrm{g}}, \nabla_{\mathrm{q}}\right\}$

The linearized equations are written as

$$
\begin{gathered}
F \nabla_{f} \aleph_{k}\left[F_{r}, G_{r}, T_{r}\right]+F \nabla_{\theta} \aleph_{k}\left[F_{r}, G_{r}, T_{r}\right]+F \nabla_{\phi} \aleph_{k}\left[F_{r}, G_{r}, T_{r}\right]= \\
F \nabla_{f}\left[F_{r}, G_{r}, T_{r}\right]+F \nabla_{\theta}\left[F_{r}, G_{r}, T_{r}\right]+F \nabla_{\phi}\left[F_{r}, G_{r}, T_{r}\right]-\aleph_{k}\left[F_{r}, G_{r}, T_{r}\right]
\end{gathered}
$$

for $k=1,2,3$ the above equations form a system of three coupled partial differential equations that can be solved iteratively for $f(x, h), g(x, h), q(x, h)$. The bivariate local-linearization scheme (BLLM) corresponding to (12) -(14) become

$$
\begin{gathered}
a_{0, r}(\xi, \eta) f_{r+1}^{\prime \prime \prime}+a_{1, r}(\xi, \eta) f_{r+1}^{\prime \prime}+a_{2, r}(\xi, \eta) f_{r+1}^{\prime}+a_{3, r}(\xi, \eta) f_{r+1}+a_{4, r}(\xi, \eta) \frac{\partial f^{\prime}{ }_{r+1}}{\partial \xi}+a_{5, r}(\xi, \eta) \frac{\partial f_{r+1}}{\partial \xi}=R_{1, r} \\
b_{0, r}(\xi, \eta) g_{r+1}^{\prime \prime}+b_{1, r}(\xi, \eta) g_{r+1}^{\prime}+b_{2, r}(\xi, \eta) g_{r+1}+b_{3, r}(\xi, \eta) \frac{\partial g_{r+1}}{\partial \xi}=R_{2, r} \\
c_{0, r}(\xi, \eta) \theta_{r+1}^{\prime \prime}+c_{1, r}(\xi, \eta) \theta_{r+1}^{\prime}+c_{2, r}(\xi, \eta) \theta_{r+1}+c_{3, r}(\xi, \eta) \frac{\partial \theta_{r+1}}{\partial \xi}=R_{3, r}
\end{gathered}
$$

Where

$$
\begin{aligned}
& a_{0, r}(\xi, \eta)=1+\epsilon_{1}\left(1-\theta_{r}\right), a_{1, r}(\xi, \eta)=(1+\xi \cot \xi) f_{r}-\epsilon_{1} \theta^{\prime}+\xi \frac{\partial f_{r}}{\partial \xi} \\
& a_{2, r}(\xi, \eta)=-\left(2{f^{\prime}}_{r}-K_{p}\left(1+\epsilon_{1}\left(1-\theta_{r}\right)\right)+\xi \frac{\partial f_{r}^{\prime}}{\partial \xi}\right) \\
& a_{3, r}(\xi, \eta)=2\left(1+\epsilon_{1}\left(1-\theta_{r}\right)\right) f_{r}, a_{4, r}(\xi, \eta)=-\xi f^{\prime}{ }_{r} a_{5, r}(\xi, \eta)=-\xi f^{\prime \prime}{ }_{r} \\
& b_{0, r}(\xi, \eta)=\left(1+\epsilon_{1}\left(1-\theta_{r}\right)\right), b_{1, r}(\xi, \eta)=(1+\xi \cot \xi) f_{r}+\xi \frac{\partial f_{r}}{\partial \xi} \\
& b_{2, r}(\xi, \eta)=-\left(2 f^{\prime}{ }_{r}-K_{p}\left(1+\epsilon_{1}\left(1-\theta_{r}\right)\right)\right), b_{3, r}(\xi, \eta)=-\xi f^{\prime}{ }_{r} \\
& c_{0, r}(\xi, \eta)=\left(1+\epsilon_{2} \theta^{\prime \prime}{ }_{r}\right), c_{1, r}(\xi, \eta)=\operatorname{Pr} f_{r}+\operatorname{Pr}(1+\xi \cot \xi) f_{r}+2 \epsilon_{2} \theta^{\prime}+\operatorname{Pr} \xi \frac{\partial f_{r}}{\partial \xi} \\
& c_{2, r}(\xi, \eta)=\left(\epsilon_{2} \theta_{r}^{\prime \prime}\right)-\operatorname{Pr} f_{r}^{\prime}-\operatorname{PrEc} \epsilon_{1}\left(f_{r}^{\prime 2}+g_{r}^{\prime 2}\right), c_{3, r}(\xi, \eta)=-\xi \operatorname{Pr} f^{\prime}{ }_{r}, \\
& R_{1, r}=\epsilon g_{r}^{2}+2\left(1+\epsilon_{1}\left(1-\theta_{r}\right)\right) f^{\prime \prime}{ }_{r} f_{r}-\theta_{r}-\xi f^{\prime}{ }_{r} \frac{\partial f_{r}}{\partial \xi} \\
& R_{2, r}=\mathbf{0} \text {, }
\end{aligned}
$$




$$
R_{3, r}=\epsilon_{2} \theta_{r}^{\prime \prime} \theta_{r}+\operatorname{Pr} \epsilon_{2} \theta_{r}^{\prime 2}-\operatorname{PrEc}\left(1+\epsilon_{1}\right)\left(f_{r}^{\prime \prime 2}+g_{r}^{\prime 2}\right)
$$

Applying the Chebyshev spectral collocation method to system (20) - (22) we obtain the numerical solutions used in this work.

\section{Results and Discussion}

In order to understand the physics of the problem, the results are illustrated in the form of Figures 1 - 6 showing the effects of various physical parameters on spin velocity profiles, velocity profiles and temperature profiles. The results are discussed in detail; we show that the method used in this work also obtains accurate results.

In this work we also present Tables 1 - 3. The tables illustrate the accuracy of the method used and displays the effect of selected parameters of the physical properties such as the skin-friction coefficient $C_{f}(x)$ and the heat-transfer coefficient $\mathrm{Nu}(\mathrm{x})$, where $\mathrm{x}$ is the time.

In this section we assume that the Prandtl number is between $\operatorname{Pr}=0.72$ at $20^{\circ} \mathrm{C}$ and $\operatorname{Pr}=1$ for Newtonian fluids. All other parameters are chosen arbitrarily being careful to stay within the acceptable range of these types of fluids.

The numerical results were validated for the skin friction $f^{\prime \prime}(0)=C_{f}(x)$ and heat transfer $-q^{\prime}(0)=N u(x)$ coefficients for the Newtonian fluid. The results obtained by the BLLM were compared to the results obtained by Ece [24] and were found to be in excellent agreement as shown in Table 1.

Table 1: Caption for table goes at the top.

\begin{tabular}{|l|lr|rc|}
\hline & \multicolumn{2}{|c|}{ Ece [24] } & \multicolumn{2}{c|}{ BLLM } \\
\hline $\operatorname{Pr}$ & $f^{\prime \prime}(0)$ & $-\theta^{\prime}(0)$ & $f^{\prime \prime}(0)$ & $-\theta^{\prime}(0)$ \\
\hline 1 & 0.681483 & 0.638855 & 0.68148323 & 0.63885528 \\
\hline 10 & 0.433268 & 1.275499 & 0.43326769 & 1.27549871 \\
\hline
\end{tabular}

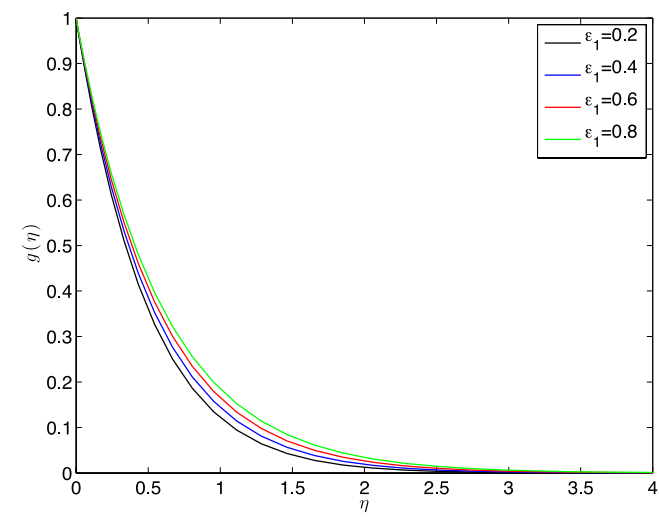

Fig. 1: Effect of $\mathrm{e}_{1}$ on spin velocity profiles for

$$
\begin{aligned}
& f_{w}=0.5, e=0.1, \operatorname{Pr}=1, E c=1, K_{p}=0.1, \\
& e_{2}=0.2 .
\end{aligned}
$$




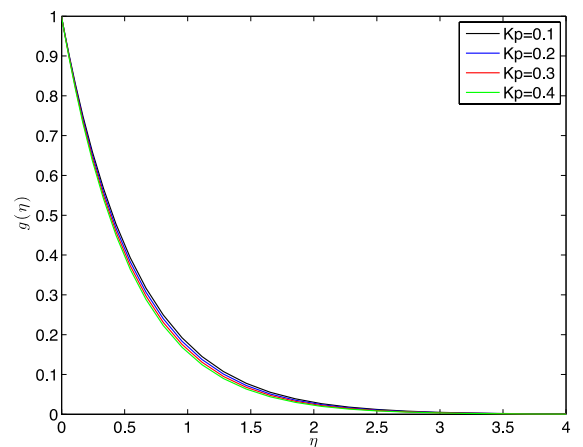

Fig. 2: Effect of $\mathrm{K}_{\mathrm{p}}$ on spin velocity profiles for

$$
\begin{aligned}
& \mathrm{f}_{\mathrm{w}}=0.5, \mathrm{e}=0.1, \operatorname{Pr}=1, \mathrm{Ec}=1, \mathrm{~K}_{\mathrm{p}}=0.1, \\
& \mathrm{e}_{2}=0.2 .
\end{aligned}
$$

In Figure 1, shows a graph of the effect of temperature-dependent viscosity parameter $\varepsilon_{1}$ on the spin velocity profiles $g(\eta)$. Increasing $\varepsilon_{1}$ result in the increase of spin velocity profiles. Increasing $\varepsilon_{1}$ reduce viscosity, therefore the velocity of flow is enhanced. Figure 2 shows the effect of the drag-force term $K p$ on spin velocity profiles. Increasing $K p$ result in the decrease in the spin velocity profiles. This is caused by the drag opposing fluid motion, which in turn reduce spinning velocity.

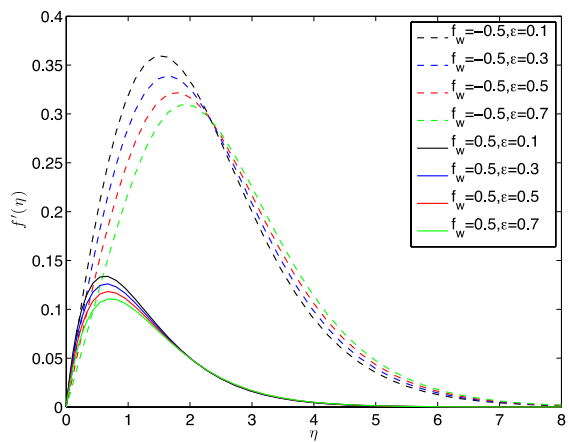

Fig. 3: Effect of e on velocity profiles for suction $f_{w}=0.5$ (suction), $f_{w}=-0.5$ (blowing) for

$$
\mathrm{e}_{1}=0.1, \operatorname{Pr}=1, \mathrm{Ec}=1, \mathrm{~K}_{\mathrm{p}}=0.1, \mathrm{e}_{2}=0.2 \text {. }
$$

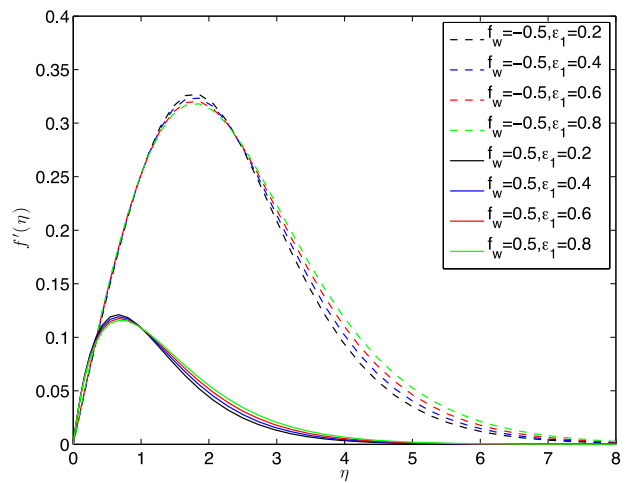

Fig. 4: Effect of $e_{1}$ on velocity profiles for suction $f_{w}=0.5$ (suction), $f_{w}=-0.5$ (blowing) for

$$
\mathrm{e}=0.1, \operatorname{Pr}=1, \mathrm{Ec}=1, \mathrm{~K}_{\mathrm{p}}=0.1, \mathrm{e}_{2}=0.2 \text {. }
$$


In Figure 3, the graph shows the effect of increasing spin parameter for both suction and blowing cases on velocity profiles $f^{\prime}(h)$. Increasing the spin parameter reduce velocity profiles. This is because the direction of fluid flow and spin direction are perpendicular, this effect then reduce velocity in the flow regime. Figure 4 shows the effect of temperature dependent viscosity $\varepsilon_{1}$ on velocity profiles $\mathrm{f}^{\prime}(\mathrm{h})$. Increasing $\varepsilon_{1}$ slightly reduce velocity profiles close to the surface and are increased further away from the surface. Increasing $\varepsilon_{1}$ reduce viscosity, the velocity close to the surface is slow due to both the spinning effect and no-slip conditions at the surface. The increase in the velocity further from the surface is attributed to the effect of spinning.

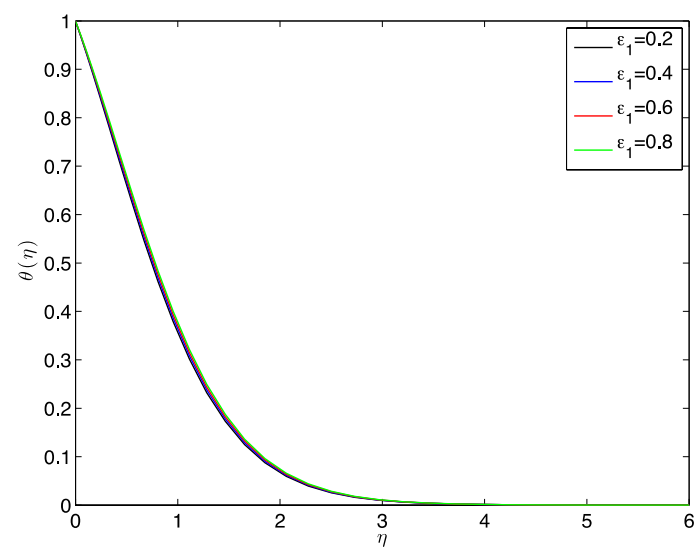

Fig. 5: Effect temperature dependent viscosity parameter on temperature profiles for $\mathrm{e}=0.1, \operatorname{Pr}=1, \mathrm{Ec}=1, \mathrm{~K}_{\mathrm{p}}=0.1, \mathrm{e}_{2}=0.2$.

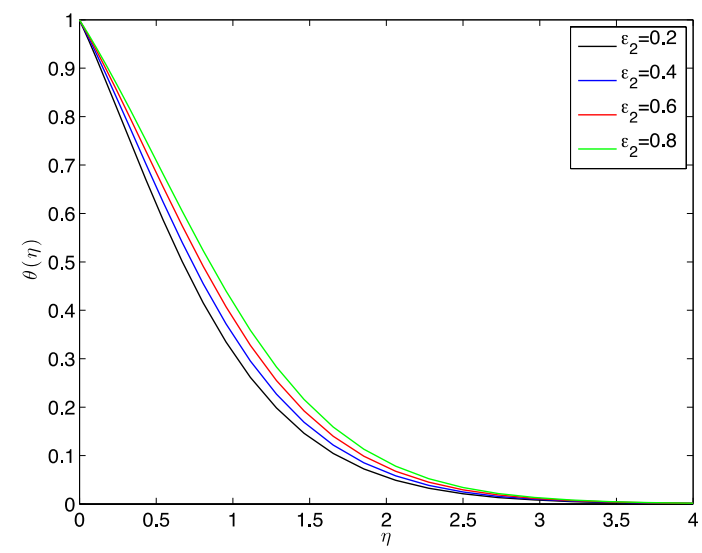

Fig. 6: Effect thermal conductivity parameter on temperature profiles for $\mathrm{e}=0.1, \operatorname{Pr}=1, \mathrm{Ec}=1, \mathrm{~K}_{\mathrm{p}}=0.1, \mathrm{e}_{1}=0.2$.

In Figure 5 shows the effect of $\varepsilon_{1}$ on temperature profiles $\mathrm{q}(\mathrm{h})$, increasing $\varepsilon_{1}$ has an effect of increasing temperature profiles. Increasing $\varepsilon_{1}$ is interpreted as increasing the temperature of a fluid which then reduce viscosity. In Figure 6 increasing $\varepsilon_{2}$ result in the increase in the temperature profiles. This effect is caused by the fact that if the heat transfer in the solid boundary is increased, this heat is then transferred through the solidliquid interface into the flow regime thereby increasing fluid temperature. 
Table 2: Effect of spin parameter $\varepsilon$ on $f^{\prime \prime}(0)$ and $-q^{\prime}(0)$ with increasing $x$.

\begin{tabular}{|l|ll|ll|ll|}
\hline & \multicolumn{2}{|c|}{$\mathrm{e}=0.1$} & \multicolumn{2}{c|}{$\mathrm{e}=0.3$} & \multicolumn{2}{c|}{$\mathrm{e}=0.5$} \\
\hline $\mathrm{x}$ & $\mathrm{f}^{\prime \prime}(0)$ & $-\mathrm{q}^{\prime}(0)$ & $\mathrm{f}^{\prime \prime}(0)$ & $-\mathrm{q}^{\prime}(0)$ & $\mathrm{f}^{\prime \prime}(0)$ & $-\mathrm{q}^{\prime}(0)$ \\
\hline 0 & 0.74468227 & 0.26467850 & 0.74468227 & 0.26467850 & 0.74468227 & 0.2646785 \\
\hline 10 & 0.74871704 & 0.88291067 & 0.80762128 & 0.88291067 & 0.68981280 & 0.88291067 \\
\hline 20 & 0.76514459 & 084807283 & 0.82341886 & 0.84807283 & 0.70687032 & 0.84807283 \\
\hline 30 & 0.82032612 & 0.90915995 & 0.75678297 & 0.90915995 & 0.69323981 & 0.90915995 \\
\hline 40 & 0.80394128 & 0.82987832 & 0.74724319 & 0.82987832 & 0.69054509 & 0.82987832 \\
\hline 50 & 0.81698621 & 0.82260666 & 0.75778304 & 0.82260666 & 0.69857987 & 0.82260666 \\
\hline 60 & 0.81515119 & 0.83760231 & 0.75349204 & 0.83760231 & 0.69183289 & 0.83760231 \\
\hline 70 & 0.79913025 & 0.78790396 & 0.74093332 & 0.78790396 & 0.68273638 & 0.78790396 \\
\hline 80 & 0.80442314 & 0.75871666 & 0.74505431 & 0.75871666 & 0.68568549 & 0.75871666 \\
\hline 90 & 0.79796829 & 0.73811823 & 0.73839852 & 0.73811823 & 0.67882875 & 0.73811823 \\
\hline 100 & 0.79231715 & 0.70837858 & 0.73309865 & 0.70837858 & 0.67388015 & 0.70837858 \\
\hline
\end{tabular}

In Table 2 shows the effect of spin parameter $\varepsilon$ on skin-friction $\mathrm{C}_{\mathrm{f}}(\mathrm{x})$ and heat- transfer $\mathrm{Nu}(\mathrm{x})$ coefficients with increasing value of $\mathrm{x}$. The skin friction coefficient increase then decreases with increasing $\mathrm{x}$. This is the skin-friction distribution around the spherical surface. The heat-transfer coefficient decrease with increasing $x$. Similarly, the heattransfer distribution around the spherical surface is shown.

Increasing the spin parameter e does not affect the skin friction coefficient for $\mathrm{x}=0$, but the distribution decreases with increasing $\mathrm{x}$. This is attributed to the fact that, increasing spin dislodges fluid contact on the surface of the sphere thereby reducing the skin-friction coefficient. Increasing the spin parameter seem not to affect the heat-transfer coefficient distribution.

Table 3: Effect of temperature-dependent viscosity parameter $\varepsilon_{1}$ on $f^{\prime \prime}(0)$ and $-q^{\prime}(0)$ with increasing $x$

\begin{tabular}{|l|ll|ll|ll|}
\hline & \multicolumn{2}{|c|}{$\mathrm{e}_{1}=0.2$} & \multicolumn{2}{c|}{$\mathrm{e}_{1}=0.4$} & \multicolumn{2}{c|}{$\mathrm{e}_{1}=0.6$} \\
\hline $\mathrm{x}$ & $\mathrm{f}^{\prime \prime}(0)$ & $-\mathrm{q}^{\prime}(0)$ & $\mathrm{f}^{\prime \prime}(0)$ & $-\mathrm{q}^{\prime}(0)$ & $\mathrm{f}^{\prime \prime}(0)$ & $-\mathrm{q}^{\prime}(0)$ \\
\hline 0 & 0.74468227 & 0.26467850 & 0.71670168 & 0.05496846 & 0.68862656 & 0.16622826 \\
\hline 10 & 0.80762128 & 0.88291067 & 0.83887649 & 0.86775129 & 0.86930503 & 0.91915083 \\
\hline 20 & 0.82341886 & 0.84807283 & 0.85441969 & 0.82265424 & 0.88462403 & 0.84747657 \\
\hline 30 & 0.82032612 & 0.90915995 & 0.85663602 & 0.90388503 & 0.89205803 & 0.96014218 \\
\hline 40 & 0.80394128 & 0.82987832 & 0.83294474 & 0.80126475 & 0.86113869 & 0.82280043 \\
\hline 50 & 0.81698621 & 0.82260666 & 0.84862801 & 0.79401796 & 0.87943995 & 0.80318861 \\
\hline 60 & 0.81515119 & 0.83760231 & 0.84941407 & 0.81611308 & 0.88278241 & 0.83243293 \\
\hline 70 & 0.79913025 & 0.78790396 & 0.82963391 & 0.75352538 & 0.85926127 & 0.74728640 \\
\hline 80 & 0.80442314 & 0.75871666 & 0.83594231 & 0.71957230 & 0.86657931 & 0.69410993 \\
\hline 90 & 0.79796829 & 0.73811823 & 0.82973575 & 0.69669319 & 0.86057312 & 0.66156762 \\
\hline 100 & 0.79231715 & 0.70837858 & 0.82353867 & 0.66519293 & 0.85381302 & 0.63345890 \\
\hline
\end{tabular}

In Table 2 shows the effect of temperature-dependent viscosity parameter $\varepsilon_{1}$ on skin friction and heat transfer coefficient with increasing value of $\xi$. Increasing $\varepsilon_{1}$ result in the increase in the skin-friction coefficient. Increasing $\varepsilon_{1}$ is interpreted as lowering the viscosity, this enhances no-slip conditions. On the contrary, if $\varepsilon_{1}$ is decreased, then viscosity increases reducing skin-friction at the surface. 
Increasing the $\varepsilon_{1}$ decrease the heat-transfer coefficient. This is caused by the fact that when the temperature is increased in the flow regime, the heat transfer on the solid-liquid interface is reduced.

\section{Conclusion}

The study of free convection from a spinning sphere with temperature-dependent viscosity, thermal conductivity and viscous dissipation effects was conducted. The most common partial differential equations which are normally solved using time consuming finite difference methods were considered. These equations were solved by recently developed spectral method called the bivariate local linearization method. This method makes use of the Chebyshev spectral collocation technique independently in both $\eta$ and $\xi$ directions. This is done by choosing Chebyshev Gauss-Lobatto points in those directions. The method is fast and easy to implement as shown the solution method section. The method avoids time consuming discretisation process and still obtains accurate results. The results obtained were compared to those in the literature and found to be in excellent agreement. This work contributes to the introduction of robust numerical methods in the area of heat and mass transfer.

The effect of selected parameters on the spin-velocity profiles is also presented. Increasing the temperature-dependent viscosity parameter increase spin-velocity profiles. Increasing the drag-force term reduce spin-velocity profiles. These results are rarely reported in the literature. This work also opens further research in more complicated partial differential equations using the numerical method used here.

\section{Acknowledgements}

I would like to thank the Department of Mathematical and Physical Sciences of the Central University of Technology and the Faculty Research and Innovation Committee (FRIC) for their financial support.

\section{References}

[1] W. J. Minkowycz, E. M. Sparrow, "Numerical solution scheme for local non similarity boundary layer analysis," Numer. Heat Transfer, vol. 1, pp. 69-85, 1978.

[2] S. S. Motsa, V. M. Magagula, P. Sibanda, "A bivariate Chebyshev spectral collocation quasi-linearization method for nonlinear evolution parabolic equations," The Scientific World Journal, Article ID 581987, p. 13, 2014, doi: $10.1155 / 2014 / 581987$

[3] R. E. Bellman, R. E. Kalaba, Quasi-linearization and nonlinear boundary value problems. Elsevier, New York, 1965.

[4] A. S. Gaffar, V. Prasad Ramachandra, O. Anwa Beg, "Numerical study of flow and heat transfer of non-Newtonian tangent hyperbolic fluid from a sphere with Biot number effects," Alexandria Engineering Journal, vol. 54, pp. 829$841,2015$.

[5] M. F. El-Amin, S. Sun, "A Finite Difference Scheme for Double-Diffusive Unsteady Free Convection from a Curved Surface to a Saturated Porous Medium with a Non-Newtonian," Fluid, Procedia Computer Science, vol. 4, pp. 948957, 2011.

[6] R. Ramteke, N. Kishore, "Heat transfer from slip spheres to a shear-thickening fluid: effects of slip velocity and particle volume fraction," Procedia Engineering, vol. 127, pp. 354-361, 2015.

[7] A. B. Rahimi and T. Jalali, "Unsteady free convection from a sphere in a porous medium with variable surface temperature," International Journal of Engineering, vol. 18, no. 4, 2005.

[8] N. Zainuddin, I. Hashim, H. Saleh, R. Roslan, "Effects of radiation on free convection from a heated horizontal circular cylinder in the presence of heat generation," Sains Malaysiana, vol. 45, no. 2, pp. 315321, 2016.

[9] F. G. Blottner, "Investigation of some finite-difference techniques for solving the boundary layer equations," Computer Methods in Applied Mechanics and Engineering, vol. 6, pp. 1-30, 1975.

[10] A. Nayela, M. Al-Juma, A. J. Chamkha, "Coupled heat and mass transfer by natural convection of a micro polar fluid flow about a sphere in porous media with Soret and Dufour effects," Recent Advances in Fluid Mechanics, Heat and Mass Transfer and Biology, ISBN:978-161804-065-7, pp. 204-209.

[11] Md. R. Haquea, Md. M. A. M. M. Ali, R. Karim, "Effects of viscous dissipation on natural convection flow over a sphere with temperature dependent thermal conductivity in presence of heat generation," Procedia Engineering vol. 105, pp. 215-224, 2015. 
[12] Md. A. Alim, Md. R. Karim, Md. M. Akand, "Heat generation effects on MHD natural convection flow along a vertical wavy surface with variable thermal conductivity," American Journal of Computational Mathematics, vol. 2, pp. 42-50, 2012.

[13] M. Narayana, P. Sibanda, S. S. Motsa1 and P. G. Siddheshwar, "On double-diffusive convection and cross diffusion effects on a horizontal wavy surface in a porous medium," Boundary Value Problems. vol. 2012, no. 88, [Online]. Available: http://www.boundaryvalueproblems.com/content/2012/1/88

[14] Z. G. Makukula, P. Sibanda, S. S. Motsa, "A novel numerical technique for two dimensional laminar flow between two moving porous walls," Mathematical Problems in Engineering, DOI:10.1155/2010/528956.

[15] S. S. Motsa and P. Sibanda, "On Extending the quasi-linearization method to higher order convergent hybrid schemes using the spectral homotopy analysis method," Journal of Applied Mathematics, vol. 2013, Article ID 879195, p. 9, http://dx.doi.org/10.1155/2013/879195.

[16] S. Nadeem, S. Saleem, "Theoretical investigation of MHD nanofluid flow over a rotating cone: An optimal solution," Inf. Sci. Lett. vol. 3, pp. 55-62, 2014.

[17] P. Bogacki, L. F. Shampine, "An efficient Runge-Kutta (4,5) pair," Computers, Mathematics and Applications, vol. 32, pp. 15-28, 1996.

[18] L. F. Shampine and P. H. Muir, "Estimating conditioning of Bvps for Odes," Mathematical and Computational Modelling, vol. 40, pp. 1309-1321, 2004.

[19] M. Ghalambaz, A. Noghrehabadi and A. Ghanbarzadeh, "Natural convection of nanofluids over a convectively heated vertical plate embedded in porous medium," Brazilian Journal of Chemical Engineering, vol. 31, no. 2, pp. 413-427.

[20] K. P. Kameswaran, M. Narayana, P. Sibanda, G. Makanda, "On radiation effects on hydromagnetic Newtonian liquid flow due to an exponentially stretching sheet," Boundary Value Problems, DOI: 10.1186/1687-2770-2012105.

[21] S. M. M. EL-Kabeir, M. A. El-Hakiem, A. M. Rashad, "Natural convection from a permeable sphere embedded in a variable porosity porous medium due to thermal dispersion," Nonlinear Analysis: Modelling and Control, vol. 12, no. 3, pp. 345357, 2007.

[22] K. Jafarpur and M. M. Yovanovich, "Laminar free convective heat transfers from isothermal spheres: a new analytical method," J. Heal Mass Transfer., vol. 35, no. 9, pp. 2195-2201, 1992.

[23] I. L. Animasaun, E. A. Adebile, A. I. Fagbade, "Casson fluid flow with variable thermo-physical property along exponentially stretching sheet with suction and exponentially decaying internal heat generation using the homotopy analysis method," Journal of the Nigerian Mathematical Society, vol. 35, pp. 117, 2016.

[24] C. M. Ece, "Free convection about a cone under mixed thermal boundary conditions and a magnetic field," Applied Mathematical Modelling, 29, 1121-1134, 2005. 